\title{
Recent developments on the KPZ surface-growth equation
}

\author{
By Horacio S. Wio ${ }^{1}$, Carlos Escudero ${ }^{2}$, Jorge A. Revelli ${ }^{1}$, \\ Roberto R. Deza ${ }^{3}$ and Marta S. De la Lama ${ }^{4}$ \\ (1) Instituto de Física de Cantabria (UC and CSIC), \\ Avda. de los Castros, s/n, E-39005 Santander, Spain; \\ (2) ICMAT (CSIC-UAM-UC3M-UCM), Departamento de Matemáticas, \\ Facultad de Ciencias, Universidad Autónoma de Madrid, \\ Ciudad Universitaria de Cantoblanco, 28049 Madrid, Spain; \\ (3) Instituto de Investigaciones Físicas Mar del Plata (UNMdP and CONICET), \\ Deán Funes 3350, B7602AYL Mar del Plata, Argentina; \\ (4) Max-Planck-Institute for Dynamics and Self-Organization, \\ Bunsenstraße 10, 37073 Göttingen, Germany
}

The stochastic nonlinear PDE known as the Kardar-Parisi-Zhang (KPZ) equation is a highly successful phenomenological mesoscopic model of surface and interface growth processes. Its suitability for analytical work, its explicit symmetries, and its prediction of an exact dynamic scaling relation for a one-dimensional (1D) substratum, led people to adopt it as a "standard" model in the field during the last quarter of century. At the same time, several conjectures deserving closer scrutiny were established as dogmas throughout the community. Among these we find the beliefs that "genuine" nonequilibrium processes are nonvariational in essence, and that the exactness of the dynamic scaling relation owes its existence to a Galilean symmetry. Additionally, the equivalence among planar and radial interface profiles has been generally assumed in the literature throughout the years. Here - among other topics - we introduce a variational formulation of the KPZ equation, remark on the importance of consistency in discretization, and challenge the mainstream view on the necessity for scaling of both Galilean symmetry and the 1D fluctuationdissipation theorem. We also derive the KPZ equation on a growing domain as a first approximation to radial growth, and outline the differences with respect to the classical case that arises in this new situation.

Keywords:

Ægrowth dynamics, Galilean invariance, variational formulation, domain growth

\section{Introduction}

Phenomena far from thermodynamic equilibrium are ubiquitous in nature, a few examples being turbulence in fluids, interface and growth problems, chemical reactions, biological systems, and economic and sociologic spatiotemporal patterns. During the last decades, statistical physics has become mature enough to shift its focus towards nonequilibrium processes. Among those studies, the understanding 
of surface-growth kinetics at microscopic and mesoscopic levels constitutes a major challenge in physics and materials science (Tong \& Williams, 1994; Barabási \& Stanley, 1995; Halpin-Healy \& Zhang, 1995; Marsili et al. , 1996). In recent papers, the methods devised for static critical phenomena have been successfully applied to nonequilibrium interface-growth phenomena, to obtain scaling properties, symmetries, the morphology of pattern formation in a driven state, etc (Hentschel, 1994; Prähofer \& Spohn, 2004; Fogedby, 2006; Ma et al. , 2007; Castro et al. , 2007).

It is a regrettable confusion enduring up today, to think that only "gradient" (or "variational") systems possess a Lyapunov function(al). This issue seemed to be already settled in standard references on pattern formation (Cross \& Hohenberg, 1993):

... For gradient systems the dynamics consists of relaxation toward the minimum in $\mathcal{F}$. This means that such functions are strictly only defined when the corresponding attractors are fixed points and there is no chaos, nor even any periodic motion at long times. As discussed further below, Eq. (3.12) can be generalized to spatially continuous systems and it provides important examples of pattern forming systems.

Graham and co-workers have introduced a "nonequilibrium potential" that is formally similar to a Lyapunov function but can be defined for an arbitrary dynamical system of the form (3.4) (Graham, 1989; Graham and Tel, 1986, 1990a,b). It is a single-valued functional in phase space $\Phi[U]$ that is constant on any attractor and decreases in any dynamics away from the attractors. It is defined formally as the solution of a complicated HamiltonianJacobi equation and has an interesting interpretation in terms of the probability distribution of the system under the influence of weak external noise (see subsection III.A.4 below). The main differences between the potential $\Phi$ and the functions $\mathcal{V}$ and $\mathcal{F}$ defined above are that (i) $\Phi$ is a highly singular function (only piecewise differentiable), and (ii) $\Phi$ does not determine the dynamics. On the contrary, it is usually necessary to know the solutions $U(t)$ in order to construct $\Phi[U]$.

Whereas it is true that a nonequilibrium system exhibiting nontrivial spatiotemporal behaviour cannot be "gradient" (or "variational") in the sense of the first paragraph in this quotation, there exists in principle (albeit hard to find) a Lyapunov function(al) for arbitrarily complex dissipative dynamics. Examples of nongradient dynamical systems for which the "nonequilibrium potential" (NEP) - that plays an analogous role as the free energy in equilibrium systems (Cross \& Hohenberg, 1993) - is known, can be found in Graham (1987); Graham \& Tél (1990); Montagne et al. (1996); Izús et al. (2009).

In a recent series of papers we have reported on the obtention of a NEP for scalar and non-scalar extended systems of the reaction-diffusion type-like activatorinhibitor systems and systems with local and nonlocal interactions - and exploited those results for the study of stochastic resonance in extended systems and other related phenomena (Wio et al. , 2002; Wio \& Deza, 2007; Wio et al. , 2009).

The Kardar-Parisi-Zhang (KPZ) (Kardar et al. , 1986; Medina et al. , 1989) equation is nowadays a paradigm as a stochastic-field description of a vast class of nonequilibrium phenomena, largely transcending the realm of surface-growth processes in which it was originally formulated (Halpin-Healy \& Zhang, 1995; 
Barabási \& Stanley, 1995). This equation, which reads

$$
\partial_{t} h(\mathbf{x}, t)=\nu \nabla^{2} h(\mathbf{x}, t)+\frac{\lambda}{2}[\nabla h(\mathbf{x}, t)]^{2}+F+\xi(\mathbf{x}, t),
$$

describes the evolution of a field $h(\mathbf{x}, t)$, corresponding to the height of a fluctuating interface on a $d$-dimensional substratum space. Here $\xi(\mathbf{x}, t)$ is a Gaussian white noise of zero mean, $\langle\xi(\mathbf{x}, t)\rangle=0$, and correlation $\left\langle\xi(\mathbf{x}, t) \xi\left(\mathbf{x}^{\prime}, t^{\prime}\right)\right\rangle=2 \varepsilon \delta\left(\mathbf{x}-\mathbf{x}^{\prime}\right) \delta(t-$ $\left.t^{\prime}\right), \nu$ is the surface tension, and $\lambda$ is proportional to the average growth velocity (it arises because the surface slope is parallel transported in the growth process).

In this paper we review some recent results we have obtained with regard to the KPZ equation. In Sec. 2 we recall some results from Wio (2009) to show how a NEP is obtained for the KPZ case; next we show how conjectures advanced by Hentschel (1994) are fulfilled, exploiting its knowledge; finally, we extend the discussion to a KPZ system including nonlocal contributions. In Sec. 3 we study the KPZ equation on a growing domain. Section 4 devotes to discretization issues, stressing the value of consistency in numerical integrations and relativizing that of the 1D fluctuationdissipation theorem and Galilean invariance. Section 5 contains the conclusions and final remarks.

\section{Nonequilibrium Potential for the KPZ equation}

(a) Derivation

We start from a general scalar reaction-diffusion equation with multiplicative noise

$$
\partial_{t} \phi(\mathbf{x}, t)=\nu \nabla^{2} \phi(\mathbf{x}, t)+\gamma \phi(\mathbf{x}, t)+\phi(\mathbf{x}, t) \eta(\mathbf{x}, t)
$$

where $\eta(\mathbf{x}, t)$ is Gaussian, white, of zero mean and intensity $\sigma$, and we assume the Stratonovich interpretation. It is known that the deterministic part of the system in Eq. (2.1) has the following NEP

$$
\mathcal{F}[\phi]=\int_{\Omega}\left\{-\frac{\gamma}{2} \phi(\mathbf{x}, t)^{2}+\frac{\nu}{2}(\nabla \phi(\mathbf{x}, t))^{2}\right\} \mathrm{d} \mathbf{x},
$$

where $\Omega$ indicates the integration range. In fact

$$
\partial_{t} \phi(\mathbf{x}, t)=-\frac{\delta \mathcal{F}[\phi]}{\delta \phi(\mathbf{x}, t)}+\phi(\mathbf{x}, t) \eta(\mathbf{x}, t)
$$

where the contribution from the boundaries is null, due to the variation $\delta \phi$ being fixed $(=0)$ at these boundaries, as usual. As shown in previous works (Wio \& Deza, 2007 ), it also fulfills the Lyapunov condition $\frac{d}{d t} \mathcal{F}[\phi] \leq 0$ (note that this condition is only strictly valid in a weak noise limit).

Exploiting the Hopf-Cole transformation we now define a new field, $h(\mathbf{x}, t)$ that, as indicated before, corresponds to an interface height,

$$
h(\mathbf{x}, t)=\frac{2 \nu}{\lambda} \ln \phi(\mathbf{x}, t)
$$

whose inverse is $\phi(\mathbf{x}, t)=\exp \left[\frac{\lambda}{2 \nu} h(\mathbf{x}, t)\right]$. Since $\phi(\mathbf{x}, t) \geq 0, h(\mathbf{x}, t)$ is always well defined. The transformed equation reads

$$
\partial_{t} h(\mathbf{x}, t)=\nu \nabla^{2} h+\frac{\lambda}{2}(\nabla h)^{2}+\frac{\lambda}{2 \nu} \gamma+\xi(\mathbf{x}, t)
$$


which is Eq. (1.1) if $F=\frac{\lambda}{2 \nu} \gamma$ and $\varepsilon=\left(\frac{\lambda}{2 \nu}\right)^{2} \sigma$. The noise term, that had a multiplicative character in Eq. (2.1), becomes additive in Eq. (1.1). If we now apply the same transformation to the NEP indicated in Eq. (2.2), we obtain

$$
\mathcal{G}[h]=\int_{\Omega} \mathrm{e}^{\frac{\lambda}{\nu} h(\mathbf{x}, t)} \frac{\lambda}{2 \nu}\left[-F+\frac{\lambda}{4}(\nabla h(\mathbf{x}, t))^{2}\right] \mathrm{d} \mathbf{x} .
$$

It is easy to prove that this functional fulfills both the relation

$$
\partial_{t} h(\mathbf{x}, t)=-\Gamma[h] \frac{\delta \mathcal{G}[h]}{\delta h(\mathbf{x}, t)}+\xi(\mathbf{x}, t)
$$

as well as the Lyapunov property $\frac{d}{d t} \mathcal{G}[h] \leq 0$, where $\Gamma[h]=\left(\frac{2 \nu}{\lambda}\right)^{2} \exp \left[-\frac{\lambda}{\nu} h(\mathbf{x}, t)\right]$. Hence we have a free-energy-like functional from which the KPZ kinetic equation can be obtained through functional derivation. Clearly, the contribution to the variation coming from the boundaries is null again.

\section{(b) Some Properties}

In Hentschel (1994), the fact that equations for relaxational self-affine surface growth are invariant under the Abelian group of global shift transformations $h(x, t) \rightarrow h(x, t)+l$, was used to bound the form of nonlinear terms and related kinetic coefficients. In Wio (2009), the relations found in Hentschel (1994) are easily seen to follow from the invariance properties of $\mathcal{G}[h]$ itself. In fact, if $l$ is an arbitrary (constant) shift,

$$
\begin{gathered}
\mathcal{G}[h+l]=K[l] \mathcal{G}[h] \quad, \quad \Gamma[h+l]=K[l]^{-1} \Gamma[h], \\
K[l]=\quad \mathrm{e}^{\frac{\lambda}{\nu} l}=\left(\frac{2 \nu}{\lambda}\right)^{2} \Gamma[l]^{-1} .
\end{gathered}
$$

In order to prove other conjectures advanced in Hentschel (1994), we introduce the free energy-like density $\widetilde{\mathcal{G}}[h, \nabla h]$, defined by $\mathcal{G}[h]=\int \mathrm{d} x \widetilde{\mathcal{G}}[h, \nabla h]$, namely

$$
\widetilde{\mathcal{G}}[h, \nabla h]=\frac{\lambda}{2 \nu} \mathrm{e}^{\frac{\lambda}{\nu} h(\mathbf{x}, t)}\left[-F+\frac{\lambda}{4}(\nabla h(\mathbf{x}, t))^{2}\right] .
$$

The relations we refer to are

$$
\widetilde{\mathcal{G}}[h, \nabla h]=\mathrm{e}^{s h} \widetilde{\mathcal{G}_{1}}\left[(\nabla h)^{2}\right] \quad, \quad \Gamma[h, \nabla h]=\mathrm{e}^{-s h} \Gamma_{1}\left[(\nabla h)^{2}\right] .
$$

According to the form of $\widetilde{\mathcal{G}}[h, \nabla h]$, the first relation above results obviously true, while for the second relation we have that $\Gamma[h, \nabla h]=\mathrm{e}^{-\operatorname{sh}(\mathbf{x}, t)} \Gamma_{0}$, where $\Gamma_{0}=1$ and $s=\frac{\lambda}{\nu}$, as $\Gamma[h]$ is not a function of $\nabla h$. Invariance under the nonlinear Galilei transformation, as discussed in Fogedby (2006), follows also from the NEP.

From the free-energy-like functional [Eq. (2.6)] for the KPZ kinetic equation and through functional differentiation, we have obtain a form [Eq. (2.7)] that resembles a (relaxation) "model A" according to the classification in Hohenberg \& Halperin (1977). However, since here the regime is far from equilibrium, its behaviour is highly nontrivial and we have no a-priori intuition to what its dynamics could be. Clearly, this is a point to keep in mind when suggesting Ansätze for the temporal behavior. 
To close this subsection we show another form of writing the NEP for the KPZ equation that was also introduced in Wio (2009). This form is

$$
\Phi[h]=\frac{\nu}{2} \int \mathrm{d} \mathbf{x}(\nabla h)^{2}-\frac{\lambda}{2} \int \mathrm{d} \mathbf{x} \int_{h_{\mathrm{ref}}}^{h(\mathbf{x})} \mathrm{d} \psi(\nabla \psi)^{2} .
$$

The variation of this functional gives

$$
\partial_{t} h(\mathbf{x}, t)=-\frac{\delta \Phi[h]}{\delta h(\mathbf{x}, t)}+\xi(\mathbf{x}, t),
$$

and the functional also fulfills the Lyapunov condition

$$
\partial_{t} \Phi[h]=-\left(\frac{\delta \Phi[h]}{\delta h(\mathbf{x}, t)}\right)^{2} \leq 0 .
$$

Hence, such a functional can be identified as the nonequilibrium potential (Graham, 1987; Graham \& Tél, 1990; Wio et al. , 2002) for the KPZ case.

\section{(c) About nonlocality}

In Wio (2009) it was shown that the functional including a nonlocal contribution (for simplicity we adopt $F=0$ ),

$$
\mathcal{G}[h]=\int_{\Omega}\left\{\left(\frac{\lambda^{2}}{8 \nu}\right)(\nabla h)^{2}+\mathrm{e}^{-\frac{\lambda}{2 \nu} h(\mathbf{x}, t)} \int_{\Omega} \mathrm{d} \mathbf{x}^{\prime} G\left(\mathbf{x}, \mathbf{x}^{\prime}\right) \mathrm{e}^{\frac{\lambda}{2 \nu} h\left(\mathbf{x}^{\prime}, t\right)}\right\} \mathrm{e}^{\frac{\lambda}{\nu} h(\mathbf{x}, t)} \mathrm{d} \mathbf{x},
$$

leads, after a functional derivation, to a generalized KPZ equation

$$
\begin{aligned}
\partial_{t} h(\mathbf{x}, t)= & \nu \nabla^{2} h(\mathbf{x}, t)+\frac{\lambda}{2}[\nabla h(\mathbf{x}, t)]^{2} \\
& -\mathrm{e}^{-\frac{\lambda}{2 \nu} h(\mathbf{x}, t)} \int_{\Omega} \mathrm{d} \mathbf{x}^{\prime} G\left(\mathbf{x}, \mathbf{x}^{\prime}\right) \mathrm{e}^{\frac{\lambda}{2 \nu} h\left(\mathbf{x}^{\prime}, t\right)}+\xi(\mathbf{x}, t) .
\end{aligned}
$$

Let us assume that the nonlocal kernel has translational invariance, that is $G\left(\mathbf{x}, \mathbf{x}^{\prime}\right)=G\left(\mathbf{x}-\mathbf{x}^{\prime}\right)$, and also, that it is of (very) "short" range. Hence, we can expand it as

$$
G\left(\mathbf{x}-\mathbf{x}^{\prime}\right)=\sum_{n=0}^{\infty} A_{2 n} \delta^{(2 n)}\left(\mathbf{x}-\mathbf{x}^{\prime}\right)
$$

with $\delta^{(n)}\left(\mathbf{x}-\mathbf{x}^{\prime}\right)=\nabla_{\mathbf{x}^{\prime}}^{n} \delta\left(\mathbf{x}-\mathbf{x}^{\prime}\right)$, and symmetry properties are taken into account. Exploiting this form of the kernel, we arrive to the following contributions in Eq. (2.13)

$$
\begin{aligned}
& \mathrm{e}^{-\frac{\lambda}{2 \nu} h(\mathbf{x}, t)} \int_{\Omega} \mathrm{d} \mathbf{x}^{\prime} G\left(\mathbf{x}-\mathbf{x}^{\prime}\right) \mathrm{e}^{\frac{\lambda}{2 \nu} h\left(\mathbf{x}^{\prime}, t\right)} \approx\left\{A_{0}+A_{2}\left[\left(\frac{\lambda}{2 \nu}\right)^{2}(\nabla h)^{2}+\frac{\lambda}{2 \nu} \nabla^{2} h\right]\right. \\
& +A_{4}\left[\left(\frac{\lambda}{2 \nu}\right)^{4}(\nabla h)^{4}+6\left(\frac{\lambda}{2 \nu}\right)^{3}(\nabla h)^{2} \nabla^{2} h+2\left(\frac{\lambda}{2 \nu}\right)^{2} \nabla^{2}(\nabla h)^{2}\right. \\
& \left.\left.-\left(\frac{\lambda}{2 \nu}\right)^{2}\left(\nabla^{2} h\right)^{2}+\frac{\lambda}{2 \nu} \nabla^{4} h\right]+A_{6} \ldots\right\}
\end{aligned}
$$


where the final dots indicate contributions of order $n \geq 3(2 n=6)$. These contributions have the same form as the ones arising in several previous works, where scaling properties, symmetry arguments, etc, have been used to discuss the possible contributions to a general form of the kinetic equation (Hentschel, 1994; Linz et al. , 2000; López et al. , 2005). Clearly, the different contributions that arose in Eq. (2.15) are tightly related to several of other previously studied equations, like the Sun-Guo-Grant equation (Sun et al. , 1989), and others (Hentschel, 1994; Castro et al. , 2007).

\section{(d) Nonlocality in $\mathrm{KPZ}$}

From the above expression we can extract a new form of NEP for the KPZ equation. Let us only retain the contribution that comes from the $n=1$ term. We define the functional

$$
\begin{aligned}
& \tilde{G}[h]=\int_{\Omega} \mathrm{d} \mathbf{x} \int_{\Omega} \mathrm{d} \mathbf{x}^{\prime} \mathrm{e}^{\frac{\lambda}{2 \nu} h(\mathbf{x}, t)} G\left(\mathbf{x}-\mathbf{x}^{\prime}\right) \mathrm{e}^{\frac{\lambda}{2 \nu} h\left(\mathbf{x}^{\prime}, t\right)}, \\
& G\left(\mathbf{x}-\mathbf{x}^{\prime}\right)=A_{2} \delta^{(2)} \cdot\left(\mathbf{x}-\mathbf{x}^{\prime}\right),
\end{aligned}
$$

The functional derivative of $\tilde{G}[h]$ yields

$$
\begin{aligned}
\frac{\delta \tilde{G}[h]}{\delta h(\mathbf{y})}= & A_{2} \frac{\lambda}{2 \nu}\left[\mathrm{e}^{\frac{\lambda}{2 \nu} h(\mathbf{y})} \int_{\Omega} \mathrm{d} \mathbf{x} \delta^{(2)}(\mathbf{y}-\mathbf{x}) \mathrm{e}^{\frac{\lambda}{2 \nu} h(\mathbf{x})}\right. \\
& \left.\quad+\mathrm{e}^{\frac{\lambda}{2 \nu} h(\mathbf{y})} \int_{\Omega} \mathrm{d} \mathbf{x} \mathrm{e}^{\frac{\lambda}{2 \nu} h(\mathbf{x})} \delta^{(2)}(\mathbf{x}-\mathbf{y})\right] \\
= & 2 A_{2} \frac{\lambda}{2 \nu} \mathrm{e}^{\frac{\lambda}{2 \nu} h(\mathbf{y})} \int_{\Omega} \mathrm{d} \mathbf{x} \delta^{(2)}(\mathbf{y}-\mathbf{x}) \mathrm{e}^{\frac{\lambda}{2 \nu} h(\mathbf{x})} \\
= & 2 A_{2} \frac{\lambda}{2 \nu} \mathrm{e}^{\frac{\lambda}{2 \nu} h(\mathbf{y})}\left[\left(\frac{\lambda}{2 \nu}\right)^{2}\left(\nabla_{\mathbf{x}} h\right)^{2}+\left(\frac{\lambda}{2 \nu}\right) \nabla_{\mathbf{y}}^{2} h\right] \mathrm{e}^{\frac{\lambda}{2 \nu} h(\mathbf{y})}
\end{aligned}
$$

Recalling that $\Gamma[h]=\left(\frac{2 \nu}{\lambda}\right)^{2} \exp \left[-\frac{\lambda}{\nu} h(\mathbf{x})\right]$, and adopting $A_{2}=-\frac{\nu}{2}$, the deterministic part of the KPZ equation results from

$$
-\Gamma[h] \frac{\delta \tilde{G}[h]}{\delta h(\mathbf{x})}=\nu \nabla_{\mathbf{x}}^{2} h+\frac{\lambda}{2}\left(\nabla_{\mathbf{x}} h\right)^{2} .
$$

The above indicated results allows us to define

$$
\begin{aligned}
\Phi_{2}[h] & =-\int \mathrm{d} \mathbf{x} \int^{h(\mathbf{x})} \mathrm{d} \psi \frac{\delta \Gamma[\psi]}{\delta \psi} \widetilde{\mathcal{G}}[\psi] \\
& =-\int \mathrm{d} \mathbf{x} \int \mathrm{d} \mathbf{x}^{\prime} \frac{2 \nu^{2}}{\lambda} \int^{h(\mathbf{x})} \mathrm{d} \psi \mathrm{e}^{\frac{-\lambda}{2 \nu} \psi(\mathbf{x})} \delta^{(2)}\left(\mathbf{x}-\mathbf{x}^{\prime}\right) \mathrm{e}^{\frac{\lambda}{2 \nu} \psi\left(\mathbf{x}^{\prime}\right)},
\end{aligned}
$$

that yields

$$
\partial_{t} h(\mathbf{x}, t)=-\frac{\delta \Phi_{2}[h]}{\delta h}+\xi(\mathbf{x}, t)
$$

Hence, $\Phi_{2}[h]$ is another representation of the NEP for KPZ. It has a very interesting form that could allow for a nice way to approximately evaluate the NEP. 


\section{The KPZ Equation on a Growing Domain}

The KPZ equation has also been related to the biologically motivated Eden model (Barabási \& Stanley, 1995). This model was introduced as a simplified probabilistic description of a developing cell colony. For long time it shows the propagation of a rough interface with an approximate radial symmetry. Numerical simulations suggested that the Eden model interface fluctuations belong to the KPZ universality class. As a first step in understanding radial interfaces (Escudero, 2008, 2009a) one can derive the KPZ equation in a growing domain (Escudero, 2009b). The simplest possibility is applying the dilatation transformation $x \rightarrow\left(t / t_{0}\right)^{\gamma} x$ to the KPZ equation to find

$$
\partial_{t} h=\nu\left(\frac{t_{0}}{t}\right)^{2 \gamma} \nabla^{2} h+\frac{\lambda}{2}\left(\frac{t_{0}}{t}\right)^{2 \gamma}(\nabla h)^{2}+\gamma F t^{\gamma-1}+\left(\frac{t_{0}}{t}\right)^{d \gamma / 2} \xi(x, t)
$$

where $\gamma>0$ is the growth index which specifies the speed with which the domain grows. Note that this equation, in absence of external fluxes (i.e. $\lambda=F=0$ ), conserves the average density; in other words, this domain growth mechanism is responsible for a simultaneous mass dilatation. It is possible to derive a KPZ equation on a growing domain in such a way that the total mass is conserved. This is achieved by introducing a dilution term, which rends the KPZ equation (Escudero, 2009b)

$$
\partial_{t} h=\nu\left(\frac{t_{0}}{t}\right)^{2 \gamma} \nabla^{2} h+\frac{\lambda}{2}\left(\frac{t_{0}}{t}\right)^{2 \gamma}(\nabla h)^{2}-\frac{d \gamma}{t} h+\gamma F t^{\gamma-1}+\left(\frac{t_{0}}{t}\right)^{d \gamma / 2} \xi(x, t)
$$

It is known that the dilution-free equation (3.1) presents memory effects which separate its behaviour from the one dictated by the Family-Vicsek scaling for large enough $\gamma$ (Escudero, 2009b). This implies a somehow paradoxical situation. As we have mentioned, there are two main symmetries associated with the $d$-dimensional KPZ equation: the Hopf-Cole transformation which maps it onto the noisy diffusion equation (Wio, 2009) and Galilean invariance which have been traditionally related to the non-renormalization of the KPZ vertex at an arbitrary order in the perturbation expansion (Medina et al. , 1989). In the case of the no-dilution KPZ equation (3.1) both symmetries are still present. Indeed, this equation transforms under the Hopf-Cole transformation $u=\exp [\lambda h /(2 \nu)]$ to

$$
\partial_{t} u=\nu\left(\frac{t_{0}}{t}\right)^{2 \gamma} \nabla^{2} u+\frac{\gamma F \lambda}{2 \nu} t^{\gamma-1} u+\frac{\lambda}{2 \nu}\left(\frac{t_{0}}{t}\right)^{d \gamma / 2} u \xi(x, t)
$$

which is again a noisy diffusion equation and it can be explicitly solved in the deterministic limit $\epsilon=0$. We find in this case

$$
u(x, t)=\frac{(1-2 \gamma)^{d / 2} \exp \left[F \lambda t^{\gamma} /(2 \nu)\right]}{\left[4 \pi t_{0}^{2 \gamma}\left(t^{1-2 \gamma}-t_{0}^{1-2 \gamma}\right)\right]^{d / 2}} \int_{\mathbb{R}^{d}} \exp \left[-\frac{|x-y|^{2}(1-2 \gamma)}{4 t_{0}^{2 \gamma}\left(t^{1-2 \gamma}-t_{0}^{1-2 \gamma}\right)}\right] u\left(y, t_{0}\right) \mathrm{d} y
$$


which corresponds to

$$
\begin{aligned}
h(x, t)= & \frac{2 \nu}{\lambda} \ln \left\{\frac{(1-2 \gamma)^{d / 2} \exp \left[F \lambda t^{\gamma} /(2 \nu)\right]}{\left[4 \pi t_{0}^{2 \gamma}\left(t^{1-2 \gamma}-t_{0}^{1-2 \gamma}\right)\right]^{d / 2}}\right\} \\
& +\frac{2 \nu}{\lambda} \ln \left\{\int_{\mathbb{R}^{d}} \exp \left[-\frac{|x-y|^{2}(1-2 \gamma)}{4 t_{0}^{2 \gamma}\left(t^{1-2 \gamma}-t_{0}^{1-2 \gamma}\right)}+\frac{\lambda}{2 \nu} h\left(y, t_{0}\right)\right] \mathrm{d} y\right\},
\end{aligned}
$$

for given initial conditions $u\left(x, t_{0}\right)$ and $h\left(x, t_{0}\right)$. If we consider the dilution KPZ Eq. (3.2) then transforming Hopf-Cole we find the nonlinear equation

$$
\partial_{t} u=\nu\left(\frac{t_{0}}{t}\right)^{2 \gamma} \nabla^{2} u-\frac{d \gamma}{t} u \ln (u)+\frac{\gamma F \lambda}{2 \nu} t^{\gamma-1} u+\frac{\lambda}{2 \nu}\left(\frac{t_{0}}{t}\right)^{d \gamma / 2} \xi(x, t) u,
$$

which may be thought of as a time dependent and spatially distributed version of the Gompertz differential equation. In this case it is not evident how to find an explicit solution at the deterministic level for an arbitrary initial condition.

As we have already explained, Galilean invariance means that the transformation indicated in Eq. (4.10), leaves the KPZ equation invariant. In case of no dilution this transformation can be replaced by

$$
x \rightarrow x-\frac{\lambda}{1-2 \gamma} v t_{0}^{2 \gamma} t^{1-2 \gamma}, \quad h \rightarrow h+v x, \quad F \rightarrow F-\frac{\lambda}{2 \gamma} v^{2} t_{0}^{2 \gamma} t^{1-3 \gamma},
$$

which leaves Eq. (3.1) invariant. If we consider dilution, then it is not clear how to extend this transformation to leave Eq. (3.2) invariant. The main difficulty comes from the dilution term which yields a non-homogeneous contribution to the dynamics as a response to the tilt transformation $h \rightarrow h+v x$. So in summary we may talk of a certain sort of Galilean invariance which is obeyed by the no-dilution KPZ dynamics (3.1) and is lost when dilution is taken into account. If it were found that the dilution equation (3.2) obeys the traditional KPZ scaling (at least in some suitable limit), then that would put into question the role that Galilean invariance has in fixing the exponents. We have already mentioned that the KPZ roughness $\alpha$ and dynamical $z$ exponents are believed to obey the scaling relation $\alpha+z=2$ in all spatial dimensions, a relation that has been traditionally attributed to Galilean invariance (Medina et al. , 1989), although this interpretation has been recently put into question (Berera \& Hochberg, 2007). Note that our numerical results on the KPZ equation precisely indicate the lack of control that Galilean invariance has on the critical exponents (Wio et al. , 2010a).

As we have already discussed, there is still another fundamental symmetry of the KPZ equation which manifests itself exclusively in one spatial dimension: the so called fluctuation-dissipation theorem. It basically says that for long times, when saturation has already being achieved, the nonlinearity ceases to be operative and the resulting interface profile would be statistically indistinguishable from that created by this equation for $\lambda \equiv 0$. For fast domain growth, we know from the linear theory that the interface never becomes correlated, and it operates, in this sense, as if it were effectively in the short time regime for all times (Escudero, 2009a,b). As a consequence, the fluctuation-dissipation theorem is not expected to play any role in this case. Of course, this result would be independent of whether we contemplated dilution or not. 


\section{Discretization Issues, Symmetry Violation and All That}

There are two main symmetries associated with the 1D KPZ equation: Galilean invariance and the fluctuation-dissipation relation. On one hand, Galilean invariance has been traditionally linked to the exactness of the relation $\alpha+z=2$ among the critical exponents, in any spatial dimensionality (the roughness exponent $\alpha$, characterizing the surface morphology in the stationary regime, and the dynamic exponent $z$, indicating the correlation length scaling as $\left.\xi(t) \sim t^{1 / z}\right)$. However, this interpretation has been criticized in this and other nonequilibrium models (Hernández-García et al. , 1993; Berera \& Hochberg, 2007). On the other hand, the second symmetry essentially tells us that in $1 \mathrm{D}$, the nonlinear (KPZ) term is not operative at long times.

Even recognizing the interesting analytical properties of the KPZ equation, it is clear that investigating the behaviour of its solutions requires the (stochastic) numerical integration of a discrete version. Such an approach has been used e.g. to obtain the critical exponents in one and more spatial dimensions (Forrest \& Toral, 1993; Beccaria \& Curci, 1994; Scalerandi et al. , 1996; Newman \& Bray, 1996; Lam \& Shin, 1998; Appert, 1999; Marinari et al. , 2000). Although a pseudospectral spatial discretization scheme has been recently introduced (Giada et al. , 2002; Gallego et al. , 2007), real-space discrete versions of Eq. (1.1) are still used for numerical simulations (Tabei et al. , 2004; Ma et al. , 2007). One reason is their relative ease of implementation and of interpretation in the case of nonhomogeneous substrates like, e.g. a quenched impurity distribution (de la Lama et al. , 2009).

\section{(a) Consistency}

We use the standard, nearest-neighbour discretization prescription as a benchmark to elucidate the constraints to be obeyed by any spatial discretization scheme, arising from the mapping between the KPZ and the diffusion equation (with multiplicative noise) through the Hopf-Cole transformation. is

The standard spatially discrete version of Eq. (2.1) (recalling that $\gamma=\lambda F / 2 \nu$ )

$$
\dot{\phi}_{j}=\frac{\nu}{a^{2}}\left(\phi_{j+1}-2 \phi_{j}+\phi_{j-1}\right)+\frac{\lambda F}{2 \nu} \phi_{j}+\frac{\lambda \varepsilon}{2 \nu} \phi_{j} \xi_{j}
$$

with $1 \leq j \leq N \equiv 0$, because of the assumed p.b.c. (the implicit sum convention is not meant in any of the discrete expressions). Here $a$ is the lattice spacing. Then, using the discrete version of Hopf-Cole transformation (Eq. (2.4))

$$
\phi_{j}(t)=\exp \left[\frac{\lambda}{2 \nu} h_{j}(t)\right]
$$

we get

$$
\dot{h}_{j}=\frac{2 \nu^{2}}{\lambda a^{2}}\left(\mathrm{e}^{\delta_{j}^{+} a}+\mathrm{e}^{\delta_{j}^{-} a}-2\right)+F+\varepsilon \xi_{j}
$$

with $\delta_{j}^{ \pm} \equiv \frac{\lambda}{2 \nu a}\left(h_{j \pm 1}-h_{j}\right)$. By expanding the exponentials up to terms of order $a^{2}$, and collecting equal powers of $a$ (observe that the zero-order contribution vanishes) we retrieve (in order to simplify we adopt $F=0$ )

$$
\dot{h}_{j}=\frac{\nu}{a^{2}}\left(h_{j+1}-2 h_{j}+h_{j-1}\right)+\frac{\lambda}{4 a^{2}}\left[\left(h_{j+1}-h_{j}\right)^{2}+\left(h_{j}-h_{j-1}\right)^{2}\right]+\varepsilon \xi_{j} .
$$


As we can see, the first and second terms on the r.h.s. of Eq. (4.4) are strictly related by virtue of Eq. (4.2). In other words, the discrete form of the Laplacian in Eq. (4.3) constrains the discrete form of the nonlinear term in the transformed equation. Latter we show again, in another way, the tight relation between the discretization of both terms. Known proposals (Lam \& Shin, 1998) fail to comply with this natural requirement.

An important feature of the Hopf-Cole transformation is that it is local, i.e. it involves neither spatial nor temporal transformations. An effect of this feature is that the discrete form of the Laplacian is the same, regardless of whether it is applied to $\phi$ or $h$.

The aforementioned criterion dictates the following discrete form for $\mathcal{F}[\phi]$ given by Eq. (2.2), thus a Lyapunov function for any finite $N$

$$
\mathcal{F}[\phi]=\frac{\nu}{2} \sum_{j=1}^{N} a\left(\left(\partial_{x} \phi\right)^{2}\right)_{j}=\frac{\nu}{4 a} \sum_{j=1}^{N}\left[\left(\phi_{j+1}-\phi_{j}\right)^{2}+\left(\phi_{j}-\phi_{j-1}\right)^{2}\right] .
$$

It is a trivial task to verify that the Laplacian is $\left(\partial_{x}^{2} \phi\right)_{j}=-a^{-1} \partial_{\phi_{j}} \mathcal{F}[\phi]$. Now, the obvious fact that this functional can also be written as $\mathcal{F}[\phi]=\frac{\nu}{2 a} \sum_{j=1}^{N}\left(\phi_{j+1}-\phi_{j}\right)^{2}$, serves to illustrate a fact that for a more elaborate discretization requires explicit calculations: the Laplacian does not uniquely determine the Lyapunov function (Wio et al. , 2010a).

\section{(b) An accurate consistent discretization}

Since the proposals of (Lam \& Shin, 1998) already involve next-to-nearest neighbors, one may seek a prescription that minimises the numerical error. An interesting choice for the Laplacian is (Abramowitz \& Stegun, 1965)

$$
\frac{1}{12 a^{2}}\left[16\left(\phi_{j+1}+\phi_{j-1}\right)-\left(\phi_{j+2}+\phi_{j-2}\right)-30 \phi_{j}\right]
$$

which has the associated discrete form for the KPZ term

$$
\begin{aligned}
\left(\partial_{x} \phi\right)^{2}= & \frac{1}{24 a^{2}}\left\{16\left[\left(\phi_{j+1}-\phi_{j}\right)^{2}+\left(\phi_{j}-\phi_{j-1}\right)^{2}\right]\right. \\
& \left.-\left[\left(\phi_{j+2}-\phi_{j}\right)^{2}+\left(\phi_{j}-\phi_{j-2}\right)^{2}\right]\right\}+\mathcal{O}\left(a^{4}\right) .
\end{aligned}
$$

Replacing this into the first line of Eq. (4.5), we obtain Eq. (4.6). Since this discretization scheme fulfills the consistency conditions, is accurate up to $\mathcal{O}\left(a^{4}\right)$ corrections, and its prescription is not more complex than other known proposals, we expect that it will be the convenient one to use when high accuracy is required in numerical schemes (Wio et al. , 2010a,b).

\section{(c) Relation with the Lyapunov functional}

In Sect. 2 we have indicated the form of the NEP for KPZ, and the way in which the functionals $\mathcal{F}[\phi]$ and $\mathcal{G}[h]$ are related (Wio, 2009). According to the previous results, we can write the discrete version of Eq. (2.6) as

$$
\mathcal{G}[h]=\frac{\lambda^{2}}{8 \nu} \frac{1}{2 a} \sum_{j} \mathrm{e}^{\frac{\lambda}{\nu} h_{j}}\left[\left(h_{j+1}-h_{j}\right)^{2}+\left(h_{j}-h_{j-1}\right)^{2}\right] .
$$


Introducing this expression into Eq. (4.4), and through a simple algebra, we obtain Eq. (4.4). This reinforces our previous result, and moreover indicates that the discrete variational formulation naturally leads to a consistent discretization of the KPZ equation.

\section{(d) The fluctuation-dissipation relation}

This relation is, together with Galilean invariance, another fundamental symmetry of the one-dimensional KPZ equation. It is clear that both symmetries are recovered when the continuum limit is taken in any reasonable discretization scheme. Thus, an accurate enough partition must yield suitable results.

The stationary probability distribution for the KPZ problem in 1D is known to be (Halpin-Healy \& Zhang, 1995; Barabási \& Stanley, 1995)

$$
\mathcal{P}_{\text {stat }}[h] \sim \exp \left\{\frac{\nu}{2 \varepsilon} \int \mathrm{d} x\left(\partial_{x} h\right)^{2}\right\} .
$$

For the discretization scheme in Eq. (4.4) (with $F=0$ ), this is

$$
\sim \exp \left\{\frac{\nu}{2 \varepsilon} \frac{1}{2 a} \sum_{j}\left[\left(h_{j+1}-h_{j}\right)^{2}+\left(h_{j}-h_{j-1}\right)^{2}\right]\right\} .
$$

Inserting this expression into the stationary Fokker-Planck equation, the only surviving term has the form

$$
\frac{1}{2 a^{3}} \sum_{j}\left[\left(h_{j+1}-h_{j}\right)^{2}+\left(h_{j}-h_{j-1}\right)^{2}\right] \times\left[h_{j+1}-2 h_{j}+h_{j-1}\right] .
$$

The continuum limit of this term is $\int \mathrm{d} x\left(\partial_{x} h\right)^{2} \partial_{x}^{2} h$, that is identically zero (HalpinHealy \& Zhang, 1995). A numerical analysis of Eq. (4.9) indicates that it is several orders of magnitude smaller than the value of the exponents' pdf [in Eq. (4.8)], and typically behaves as $\mathcal{O}(1 / N)$, where $N$ is the number of spatial points used in the discretization. Moreover, it shows an even faster approach to zero if expressions with higher accuracy [like Eqs. (4.6) and (4.7)] are used for the differential operators. In addition, when the discrete form of $\left(\partial_{x} h\right)^{2}$ from Lam \& Shin (1998) is used together with its consistent form for the Laplacian, the fluctuation-dissipation relation is not exactly fulfilled. This indicates that the problem with the fluctuation-dissipation theorem in $1+1$, discussed in Lam \& Shin (1998); Giada et al. (2002) can be just circumvented by using more accurate expressions.

\section{(e) Galilean invariance}

This invariance means that the transformation

$$
x \rightarrow x-\lambda v t \quad, \quad h \rightarrow h+v x \quad, \quad F \rightarrow F-\frac{\lambda}{2} v^{2},
$$

where $v$ is an arbitrary constant vector field, leaves the KPZ equation invariant. The equation obtained using the classical discretization

$$
\partial_{x} h \rightarrow \frac{1}{2 a}\left(h_{j+1}-h_{j-1}\right),
$$


is invariant under the discrete Galilean transformation

$$
j a \rightarrow j a-\lambda v t, \quad h_{j} \rightarrow h_{j}+v j a, \quad F \rightarrow F-\frac{\lambda}{2} v^{2} .
$$

However, the associated equation is known to be numerically unstable (Newman \& Bray, 1996), at least when $a$ is not small enough. Besides, Eq. (4.4) is not invariant under the discrete Galilean transformation. In fact, the transformation $h \rightarrow h+v j a$ yields an excess term which is compatible with the gradient discretization in Eq. (4.11); however this discretization does not allow to recover the quadratic term in Eq. (4.4), indicating that this finite-difference scheme is not Galilean-invariant.

Since Eq. (4.1) is invariant under the transformation indicated in Eq. (4.12), it is the nonlinear Hopf-Cole transformation (within the present discrete context) which is responsible for the loss of Galilean invariance. Note that these results are independent of whether we consider this discretization scheme or a more accurate one.

Galilean invariance has always been associated with the exactness of the onedimensional KPZ exponents, and with a relation that connects the critical exponents in higher dimensions (Medina et al. , 1989). If the numerical solution obtained from a finite-difference scheme as Eq. (4.4), which is not Galilean invariant, yields the well known critical exponents, that would strongly suggest that Galilean invariance is not a fundamental symmetry as usually considered. The numerical results presented in Wio et al. (2010a,b) clearly show that this is the case.

The moral from the present analysis is clear: due to the locality of the Hopf-Cole transformation the discrete forms of the Laplacian and the nonlinear (KPZ) term cannot be chosen independently; moreover, the prescriptions should be the same, regardless of the fields they are applied to. Equation (4.3) has also been written in (Newman \& Bray, 1996), although with different goals than ours (their interest was to analyze the strong coupling limit via mapping to the directed polymer problem).

\section{Conclusions}

The present work shortly reviews some new results in the study and analysis of the KPZ equation, that offer an alternative approach to other well known techniques. We have here found the form of the Lyapunov functional or nonequilibrium potential for the KPZ equation, and have also devised a way to extend the procedure to derive it. From this NEP, and through a functional derivative, we have obtained the KPZ equation and have also shown that such a NEP fulfills global shift properties, as well as other ones anticipated for such an unknown functional (Hentschel, 1994).

Dynamic renormalization group techniques, being useful and powerful, in many cases only offer incomplete results, having no access to the strong coupling phase (Barabási \& Stanley, 1995). Hence, it is clear the need of alternative ways to analyze the KPZ and related problems, as for instance the self-consistent expansion (Katzav, 2003). The present results open new possibilities for non-perturbational studies of the KPZ problem.

As a first approach to the understanding of radial growth we have introduced the KPZ equation on a growing domain. This is justified on the grounds that the widespread Eden model, which is related to both biological growth and percolation, belongs to the KPZ universality class. It has been traditionally accepted that radial 
and planar interfaces behave analogously. However we have seen that there are two natural ways of extending the KPZ equation to the growing domain setting. One causes the simultaneous growth of mass together with space, and the other keeps mass constant as the space dilates. The first of these generates memory effects which separate the interface fluctuations from the behavior dictated by the Family-Vicsek ansatz, while the second is free from these memory effects. On the other hand, the first approach respects the Galilean invariance symmetry and the Hopf-Cole transformation to a linear equation, while the second one does not. This again suggests that some of the mathematical properties of the classical KPZ equation, which were intuitively related to the scaling of the surface fluctuations, are not necessary in order to define the universality class.

We have also discussed the implications of the NEP in the obtention of consistent discrete representations of the KPZ equation. The fact that the KPZ equation is the result of a local transformation from a stochastic partial differential equation with multiplicative noise imposes some constraints in the discrete versions of the equation. A major point has been to call the attention to the true role of Galilean invariance in KPZ (Wio et al. , 2010a,b). Our purpose was not to compare alternative spatial discretization schemes with regard to specific KPZ features, nor to present concrete results concerning the violation of Galilean invariance. The consequences of such an analysis are general, as in obtaining the discrete versions of any set of differential equations related through a local transformation, both the original (or leading) equation and the transformation rules should be taken into account.

Regarding the recently introduced pseudospectral (PS) approach (Giada et al. , 2002; Gallego et al. , 2007) it has already been said that, when analyzing situations where defects or impurities are present, such methods do not apply and one must resort to real-space discrete forms of the differential operators (de la Lama et al. , 2009; Ramasco et al. , 2006). This drawback aside, it has advantages. One of them is related to the numerical instabilities in discrete growth models: whereas in (Dasgupta et al. , 1997), the problem has been tackled by introducing higher order contributions, in PS treatments of the same problem such an instability seems not to arise (or at least is delayed). In addition, the PS approach seems to be in principle "transparent" to the question of consistency. Nonetheless, in a forthcoming paper (Wio et al. , 2010b) we establish a relation between the real-space discretization schemes here discussed (of which more details are given) and the PS methods, in the limit where - in order to define a highly accurate discrete representation of the differential operators - we use all the $N$ lattice points.

The authors thank R. Cuerno, H. Fogedby, J.M. López and M.A. Rodríguez, for fruitful discussions and/or valuable comments. HSW and JAR acknowledge financial support from MEC, Spain, through Project CGL2007-64387/CLI. RRD acknowledges financial support from CONICET and UNMdP of Argentina. CE acknowledges financial support from the MICINN, Spain, through Project No. MTM2008-03754. The international collaboration has been facilitated by AECID, Spain, through Projects A/013666/07 and A/018685/08.

\section{References}

Abramowitz, M., \& Stegun, I. A. 1965. Handbook of Mathematical Functions: with Formulas, Graphs, and Mathematical Tables, pp. 884. New York: Dover. 
Appert, C. 1999. Universality of the growth velocity distribution in $1+1$ dimensional growth models. Comp. Phys. Comm., 121-122, 363-365. (DOI 10.1016/S00104655(99)00354-9.).

Barabási, A.-L., \& Stanley, H. E. 1995. Fractal concepts in surface growth. Cambridge University Press.

Beccaria, M., \& Curci, G. 1994. Numerical simulation of the Kardar-Parisi-Zhang equation. Phys. Rev. E, 50, 4560-4563. (DOI 10.1103/PhysRevE.50.4560.).

Berera, A., \& Hochberg, D. 2007. Gauge Symmetry and Slavnov-Taylor Identities for Randomly Stirred Fluids. Phys. Rev. Lett., 99, 254501 (1-4). (DOI 10.1103/PhysRevLett.99.254501.).

Castro, M., Muñoz-García, J., Cuerno, R., García-Hernández, M. M., \& Vázquez, L. 2007. Generic equations for pattern formation in evolving interfaces. New J. Phys., 9, 102 (1-12). (DOI 10.1088/1367-2630/9/4/102.).

Cross, M. C., \& Hohenberg, P. C. 1993. Pattern formation outside of equilibrium. Rev. Mod. Phys., 65, 851-1112. (DOI 10.1103/RevModPhys.65.851. See particularly p. 868.).

Dasgupta, C., Kim, J. M., Dutta, M., \& Das Sarma, S. 1997. Instability, intermittency, and multiscaling in discrete growth models of kinetic roughening. Phys. Rev. E, 55, 2235. (DOI 10.1103/PhysRevE.55.2235.).

de la Lama, M. S., López, J. M., Ramasco, J. J., \& Rodríguez, M. A. 2009. Activity statistics of a forced elastic string in a disordered medium. JSTAT, P07009. (DOI 10.1088/1742-5468/2009/07/P07009.).

Escudero, C. 2008. Dynamic scaling of non-Euclidean interfaces. Phys. Rev. Lett., 100, 116101 (1-4). (DOI 10.1103/PhysRevLett.100.116101.).

Escudero, C. 2009a. Dynamics of Curved Interfaces. Ann. Phys., 324, 1796-1814. (DOI 10.1016/j.aop.2009.05.003.).

Escudero, C. 2009b. Stochastic growth equations on growing domains. JSTAT, P07020. (DOI 10.1088/1742-5468/2009/07/P07020.).

Fogedby, H. C. 2006. Kardar-Parisi-Zhang equation in the weak noise limit: Pattern formation and upper critical dimension. Phys. Rev. E, 73, 031104 (1-26). (DOI 10.1103/PhysRevE.73.031104.).

Forrest, B. M., \& Toral, R. 1993. Crossover and finite size effects in the $(1+1)$ dimensional Kardar-Parisi-Zhang equation. J. Stat. Phys., 70, 703-720. (DOI 10.1007/BF01053591.).

Gallego, R., Castro, M., \& López, J. M. 2007. Pseudospectral versus finite-difference schemes in the numerical integration of stochastic models of surface growth. Phys. Rev. E, 76, 051121. (DOI 10.1103/PhysRevE.76.051121.).

Giada, L., Giacometti, A., \& Rossi, M. 2002. Pseudospectral method for the KardarParisi-Zhang equation. Phys. Rev. E, 65, 036134. (DOI 10.1103/PhysRevE.65.036134.).

Graham, R. 1987. Weak Noise Limit and Nonequilibrium Potentials of Dissipative Dynamical Systems. In Instabilities and Nonequilibrium Structures (ed. E. Tirapegui \& D. Villaroel), pp. 271-290. Dordrecht: D. Reidel. 
Graham, R., \& Tél, T. 1990. Steady-state ensemble for the complex Ginzburg-Landau equation with weak noise. Phys. Rev. A, 42, 4661-4667. (DOI 10.1103/PhysRevA.42.4661.).

Halpin-Healy, T., \& Zhang, Y.-C. 1995. Kinetic roughening phenomena, stochastic growth, directed polymers and all that. Aspects of multidisciplinary statistical mechanics. Phys. Rep., 254, 215-414. (DOI 10.1016/0370-1573(94)00087-J.).

Hentschel, H. G. E. 1994. Shift invariance and surface growth. J. Phys. A: Math. Gen., 27, 2269-2275. (DOI 10.1088/0305-4470/27/7/008.).

Hernández-García, E., Ala-Nissila, T., \& Grant, M. 1993. Interface roughening with a time-varying external driving force. Europhys. Lett., 21, 401-406. (DOI 10.1209/02955075/21/4/004.).

Hohenberg, P. C., \& Halperin, B. 1977. Theory of dynamic critical phenomena. Rev. Mod. Phys., 49, 435-479. (DOI 10.1103/RevModPhys.49.435.).

Izús, G. G., Sánchez, A. D., \& Deza, R. R. 2009. Noise-driven synchronization of a FitzHugh-Nagumo ring with phase-repulsive coupling: A perspective from the system's nonequilibrium potential. Physica A, 388, 967-976. (DOI 10.1016/j.physa.2008.11.031.).

Kardar, M., Parisi, G., \& Zhang, Y.-C. 1986. Dynamic Scaling of Growing Interfaces. Phys. Rev. Lett., 56, 889-892. (DOI 10.1103/PhysRevLett.56.889.).

Katzav, E. 2003. Self-consistent expansion results for the nonlocal Kardar-Parisi-Zhang equation. Phys. Rev. E, 68, 046113 (1-8). (DOI 10.1103/PhysRevE.68.046113.).

Lam, C.-H., \& Shin, F. G. 1998. Improved discretization of the Kardar-Parisi-Zhang equation. Phys. Rev. E, 58, 5592-5595. (DOI 10.1103/PhysRevE.58.5592.).

Linz, S. J., Raible, M., \& Hänggi, P. 2000. . In Stochastic Processes in Physics (ed. J. A. Freund \& T. Pöschel), LNP 557, pp. 473-483. Berlin: Springer.

López, J. M., Castro, M., \& Gallego, R. 2005. Scaling of Local Slopes, Conservation Laws, and Anomalous Roughening in Surface Growth. Phys. Rev. Lett., 94, 166103 (1-4). (DOI 10.1103/PhysRevLett.94.166103.).

Ma, K., Jiang, J., \& Yang, C. B. 2007. Scaling behavior of roughness in the two-dimensional Kardar-Parisi-Zhang growth. Physica A, 378, 194-200. (DOI 10.1016/j.physa.2006.12.010.).

Marinari, E., Pagnani, A., \& Parisi, G. 2000. Critical exponents of the KPZ equation via multi-surface coding numerical simulations. J. Phys. A: Math. Gen., 33, 8181-8192. (doi: 10.1088/0305-4470/33/46/303.).

Marsili, M., Maritan, A., Toigo, F., \& Banavar, J. R. 1996. Stochastic growth equations and reparametrization invariance. Rev. Mod. Phys., 68, 963-983. (DOI 10.1103/RevModPhys.68.963.).

Medina, E., Hwa, T., Kardar, M., \& Zhang, Y-C. 1989. Burgers equation with correlated noise: Renormalization-group analysis and applications to directed polymers and interface growth. Phys. Rev. A, 39, 3053-3075. (DOI 10.1103/PhysRevA.39.3053.). 
Montagne, R., Hernández-García, E., \& San Miguel, M. 1996. Numerical study of a Lyapunov functional for the complex Ginzburg-Landau equation. Physica D, 96, 4765. (DOI 10.1016/0167-2789(96)00013-9.).

Newman, T. J., \& Bray, A. J. 1996. Strong-coupling behaviour in discrete KardarParisi-Zhang equations. J. Phys. A: Math. Gen., 29, 7917-7928. (DOI 10.1088/03054470/29/24/016.).

Prähofer, M., \& Spohn, H. 2004. Exact Scaling Functions for OneDimensional Stationary KPZ Growth. J. Stat. Phys., 115, 255-279. (DOI 10.1023/B:JOSS.0000019810.21828.fc.).

Ramasco, J. J., López, J. M., \& Rodríguez, M. A. 2006. Glassy dynamics, aging, and temperature-induced avalanches in interface pinning at finite temperatures. Europhys. Lett., 76, 554-560. (DOI 10.1209/epl/i2006-10316-1.).

Scalerandi, M., Delsanto, P. P., \& Biancotto, S. 1996. Time evolution of growth phenomena in the KPZ model. Comp. Phys. Comm., 97, 195-198. (DOI 10.1016/00104655(96)00037-9.).

Sun, T., Guo, H., \& Grant, M. 1989. Dynamics of driven interfaces with a conservation law. Phys. Rev. A, 40, 6763-6766. (DOI 10.1103/PhysRevA.40.6763.).

Tabei, S. M. A., Bahraminasab, A., Masoudi, A. A., Mousavi, S. S., \& Reza Rahimi Tabar, M. 2004. Intermittency of height fluctuations in stationary state of the Kardar-ParisiZhang equation with infinitesimal surface tension in $1+1$ dimensions. Phys. Rev. E, 70, 031101. (DOI 10.1103/PhysRevE.70.031101.).

Tong, W. M., \& Williams, R. S. 1994. Kinetics of Surface Growth: Phenomenology, Scaling, and Mechanisms of Smoothening and Roughening. Annu. Rev. Phys. Chem., 45, 401-438. (DOI 10.1146/annurev.pc.45.100194.002153.).

Wio, H. S. 2009. Variational Formulation for the KPZ and Related Kinetic Equations. Int. J. Bif. Chaos, 19, 2813-2821. (DOI 10.1142/S0218127409024505.).

Wio, H. S., \& Deza, R. R. 2007. Aspects of stochastic resonance in reaction-diffusion systems: The nonequilibrium-potential approach. Europ. Phys. J.-Special Topics, 146, 111-126. (DOI 10.1140/epjst/e2007-00173-0.).

Wio, H. S., Bouzat, S., \& von Haeften, B. 2002. Stochastic Resonance in Spatially Extended Systems: the Role of Far From Equilibrium Potentials. Physica A, 306, 140-156. (DOI 10.1016/S0378-4371(02)00493-4.).

Wio, H. S., Revelli, J. A., Rodríguez, M. A., Deza, R. R., \& Izús, G. G. 2009. Stochastic Resonance in Extended Systems: An Overview of Recent Results for Systems with and without Nonequilibrium Potential. Europ. Phys. J. B, 69, 71-80. (DOI 10.1140/epjb/e2009-00071-1.).

Wio, H. S., Revelli, J. A., Deza, R. R., Escudero, C., \& de La Lama, M. S. 2010a. -KPZ equation: Galilean-invariance violation, consistency, and fluctuation-dissipation issues in real-space discretization. Europhys. Lett. (in press.).

Wio, H. S., Revelli, J. A., Deza, R. R., Escudero, C., \& de La Lama, M. S. 2010b. Discretization-related issues in the KPZ equation: Consistency, Galileaninvariance violation, and fluctuation-dissipation relation. Phys. Rev. E. (submitted.) arXiv:1001.2351v1. 